\title{
Mechanisms of Stress Generation and Relaxation in Thin Films and Coatings
}

\author{
Artur R. Shugurov ${ }^{1, \text { a) }}$ and Alexey V. Panin ${ }^{1, b)}$ \\ ${ }^{1}$ Institute of Strength Physics and Materials Science SB RAS, Tomsk, 634055, Russia

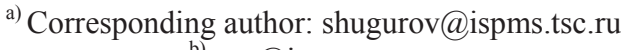 \\ b) pav@ispms.tsc.ru
}

\begin{abstract}
The failure modes of thin metal films and ceramic coatings induced by both intrinsic (growth) stresses and external effects (uniaxial tension, alternating bending, thermal treatment) were studied. Stress relaxation that causes soft films and hard coatings to fail was demonstrated to be governed by essentially the same mechanisms. The primary factor governing the reliability of thin-film structures was shown to be the strength of the film/substrate interface.
\end{abstract}

Keywords: thin films, coatings, stresses and strains, interface, cracking, buckling

\section{INTRODUCTION}

The issue of stress-induced failure phenomena in thin films and coatings is of crucial importance due to their extensive applications in microelectronics, optics, biotechnology, micromechanics, aerospace and tool industries, etc. Film cracking and spalling can be induced by tensile stress, while buckling and delamination result from relaxation of compressive stress [1]. Understanding of competing mechanisms of stress generation and relaxation is necessary to control the stress-strain state of thin-film structures and improve their reliability.

The stresses generated in thin films and coatings can be induced by external factors such as thermal or mechanical effects (extrinsic stress) as well as by evolution of their structure and phase composition which occurs during film growth (intrinsic or growth stress). In contrast to intrinsic stress that results from contributions of a number of mechanisms, some of which are still not well understood, extrinsic stress can be studied in wellcontrolled experimental conditions. Thus, this paper is focused on comparison of failure mechanisms of thin metal films and ceramic coatings induced by intrinsic and extrinsic stresses to attain revealing their common nature and understanding factors governing reliability of thin-film structures.

\section{GENERATION OF INTRINSIC STRESSES AND RELATED FILM FAILURE MODES \\ Mechanisms of Arising Intrinsic Stresses in Thin Films and Coatings}

Depending on the deposited material and the deposition parameters, intrinsic stresses that develop in thin films and coatings can be tensile or compressive. The generation of tensile stress in growing polycrystalline films is usually considered to result from island coalescence and grain boundary formation [2]. Another origin of the tensile stress is film densification due to its recrystallization and redistribution of excess free volume associated with grain boundaries, voids, vacancies, etc. [3]. In turn, generation of compressive stress has been attributed to the effect of the Laplace pressure caused by surface tension of islands before their coalescence [4], interactions of adatoms with the growing surface [5], flux-driven incorporation of excess atoms in grain boundaries [6] or between neighboring surface ledges [7]. An important mechanism of arising compressive stress in sputter-deposited films and coatings is atomic peening, i.e. incorporation of interstitials and misfitting atoms into a film due to bombardment of energetic particles [8]. In addition, intrinsic stress in thin films can be due to phase transformations, precipitation, incorporation of impurities, absorption and desorption of moisture and volatile species, etc. [1,9].

International Conference on Physical Mesomechanics of Multilevel Systems 2014

AIP Conf. Proc. 1623, 575-578 (2014); doi: 10.1063/1.4899010

(C) 2014 AIP Publishing LLC 978-0-7354-1260-6/\$30.00 




(a)

(b)

(c)

FIGURE 1. Failure modes of film films on substrates: (a) cracking; (b) edge delamination; (c) buckle delamination

Because of the rigid bonding between the film and the substrate at the interface, intrinsic stresses in thin films usually cause the film/substrate system to bend that allows their partial relaxation. The film stresses are tensile, when the substrate is bent concave upward, and compressive in the opposite case. The corresponding shear stresses arise at the film/substrate interface because of the difference in elastic characteristics of the film and the substrate.

\section{Failure Modes of Thin Films Induced by Intrinsic Stresses}

Failure modes of thin films and coatings induced by intrinsic stresses are governed by the correlation between their strength and the shear strength of the interface. When in-plane tensile stresses exceed the fracture strength of the film, it fails via the formation of multiple cracks (Fig. 1(a)). Due to their low elastic modulus and high plasticity, cracking is rather seldom in thin metal films, however, it is common for brittle ceramic films and coatings. This is clearly evident in Fig. 2(a) for a $\mathrm{SiC}_{x} \mathrm{~N}_{y}$ film grown by plasma-enhanced chemical vapor deposition on the silicon substrate. Because the coefficient of thermal expansion of the silicon carbonitride film is close to that of the silicon substrate, it appears that the cracking is induced by the growth stress rather than the thermal one. If the shear stresses become higher than the ultimate shear strength of the interface, film delamination occurs. In the case of tensile stress, delamination initiates predominantly at the edges of a film, where the interface shear stress attains maximum values (Fig. 1(b)). The debond flaws with typical size being approximately equal to the film thickness $h_{\mathrm{f}}$ are necessary to originate edge delamination [10].

Two of the primary failure modes for compressed films are edge delamination and buckle delamination (Fig. 1(c)). The latter mode is more common, although it needs pre-existing flaws at the interface with typical size of about $20 h_{\mathrm{f}}$. A film buckles after exceeding a critical stress value that is proportional to its elastic modulus and thickness and inversely proportional to the size of the debond flaw at the interface $2 b$. Therefore, buckling is more common for thin metal films; while ceramic coatings, which have high bending energy, can buckle only in the case of very small thickness or large debond flaws. Under biaxial strain, circular buckles are usually observed (Fig. 2(b)), although stripe or phone cord-like buckle structures can be formed too (Fig. 2(c)). They result from non-uniform stress-strain state of the film in the vicinity of buckling area. Compressive stresses relax in the buckling region, however, along its boundary the film stays bonded to the substrate that causes high stress gradients to appear under transition from the relaxed to unrelaxed areas. The high stress gradients govern further film delamination and corresponding form change of the buckling region.
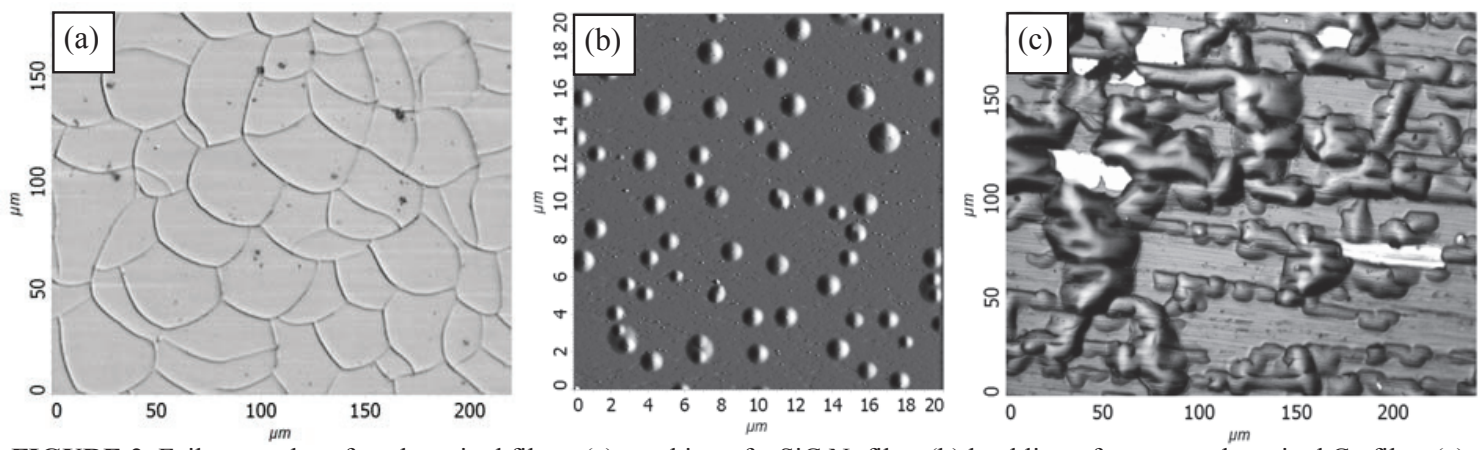

FIGURE 2. Failure modes of as-deposited films: (a) cracking of a $\mathrm{SiC}_{x} \mathrm{~N}_{y}$ film; (b) buckling of a sputter-deposited Cu film; (c) buckling and peeling of a sputter-deposited $\mathrm{Si}-\mathrm{Al}-\mathrm{N}$ coating. Optical (a, c) and atomic force microscopy (b) 

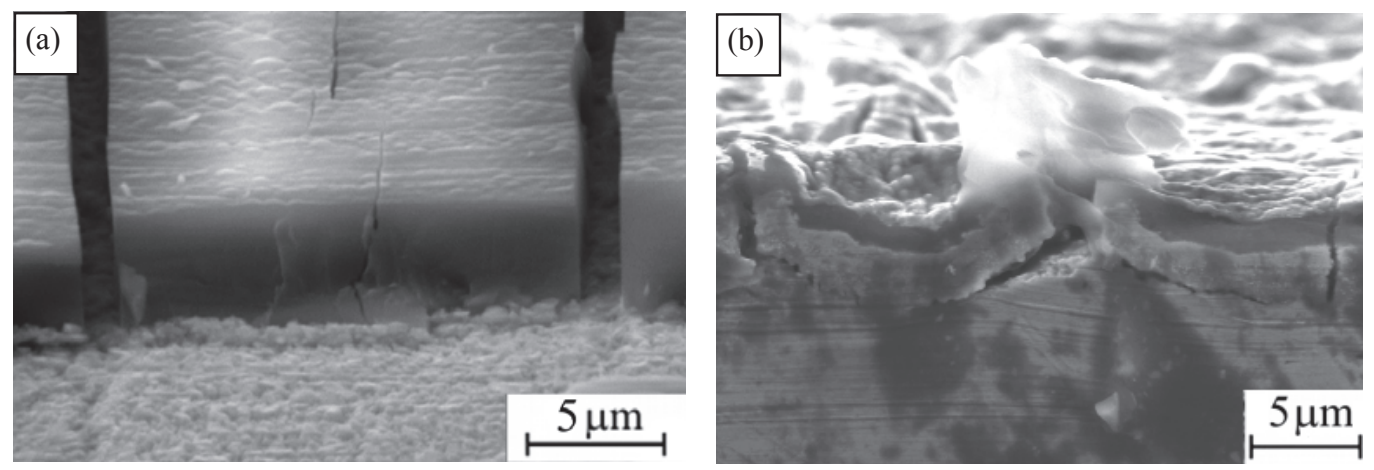

FIGURE 3. Fracture of $\mathrm{Si}-\mathrm{Al}-\mathrm{N}$ coatings subjected to uniaxial tension: (a) cracking without delamination;

(b) cracking with edge delamination of fragments. Scanning electron microscopy

\section{FAILURE OF THIN FILMS AND COATINGS SUBJECTED TO EXTERNAL EFFECTS}

Stress-induced failure of thin films and coatings was studied in details under uniaxial tension, alternating bending and thermal cycling, which loading conditions are assumed to be similar that of common applications of thin-film structures. As with relaxation of an intrinsic tensile stress, the failure of films and coatings subjected to uniaxial tension occurs by means of edge delamination or formation of transverse cracks. In the latter case, separate film fragments between neighboring cracks are strained independently on the following loading. The stress distribution in the fragments is similar to that in initial continuous coating: in-plane tensile stresses are maximal at the center of a fragment and decrease rapidly to zero near a crack, while the interface shear stresses are zero at the center and attain a maximum value close to the edge. Accordingly, as the strain increases, the competition of the fracture mechanisms can lead to either new transverse cracks in the center of a coating fragment (mode I cracking, see Fig. 3(a)) or to edge delamination of the fragment (mode II cracking, Fig. 3(b)). Transverse cracking effectively relaxes the in-plane stresses; therefore, a higher strain increment is required for arising new cracks in decreasing coating fragments. As a result, the shear stresses at the coating/substrate interface increase, which relaxation occurs by the formation of mode II cracks along fragment edges and can lead to spalling of coating fragments. Therefore, a smaller spacing between neighbor transverse cracks indicates a better adhesion of the coating to the substrate. Similar failure modes were observed in the case of biaxial tension of films induced by means of thermal excursions.

As in the case of intrinsic compressive stress, the primary mechanism of relaxation of extrinsic compression in thin films and coatings is their buckle delamination. When a film is deposited on a rigid substrate, which bending deformation is severely limited, stress-induced buckling occurs in the areas where bonding between the film and the substrate is locally weakened by various interfacial defects.
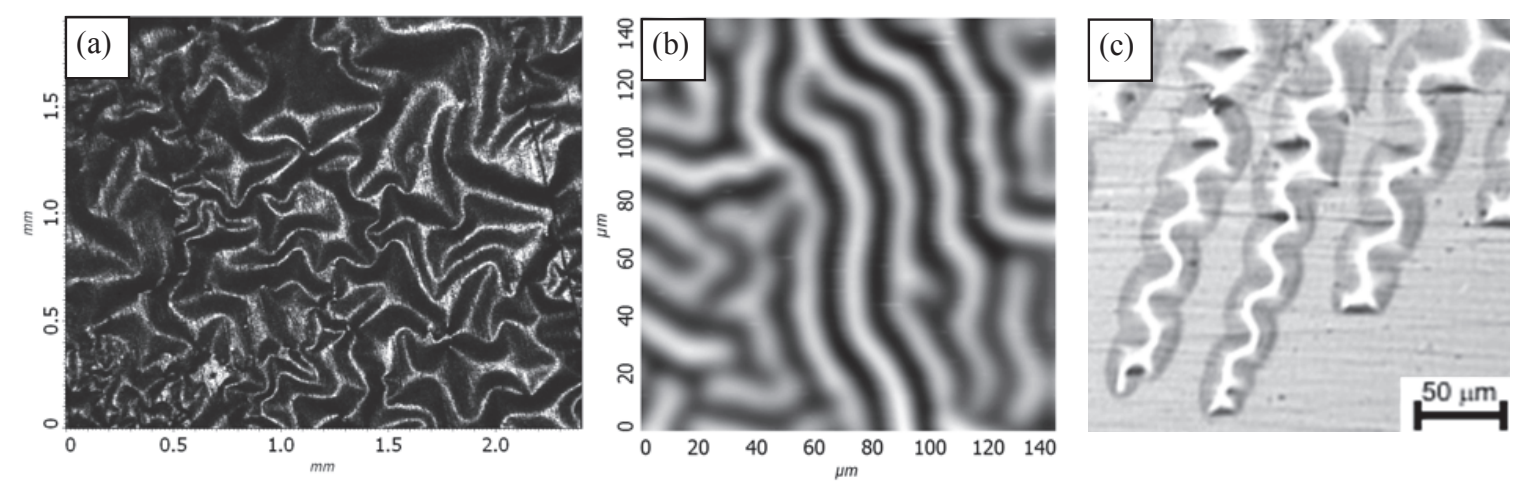

FIGURE 4. Buckling modes of thin films: (a) buckling of a copper oxide film thermally grown on a commercial copper substrate; (b) coherent wrinkling of an $\mathrm{Al} /$ polystyrene bilayer film on a Si substrate after annealing at $110^{\circ} \mathrm{C}$;

(c) wrinkling-induced buckling of a Ti film deposited on an $\mathrm{Al}$ substrate after alternating bending tests. Optical (a, c) and atomic force microscopy (b) 
As a result, buckle areas are randomly distributed over the film surface (Fig. 4(a)). A substantially other buckling mode is observed under compression of elastic films on rather compliant substrate. In this case, relaxation of compressive stresses initiates by means of coherent wrinkling of the film/substrate system, with wrinkles being periodically distributed at least in the local areas (Fig. 4(b)). If the two initial in-plane stress components are equal, then disordered labyrinth or partially ordered herringbone patterns can be formed. When the in-plane stress components are unequal (including both uniaxial and biaxial compression), initial wrinkling is always onedimensional with crests lying perpendicular to the direction of maximum compression. The local curvature of the interface produces a bending moment giving rise to appearance of tensile stress component at the wrinkle crests directed along the normal to the film plane. The tensile stress relaxation results in formation of the zones of local film debonding from the substrate at the crests of wrinkles. This implies periodically distributed secondary buckling which pattern inherits the primary wrinkling structure (Fig. 4(c)).

\section{SUMMARY}

The key role of the film/substrate interface in the failure of thin-film structures induced by relaxation of intrinsic and extrinsic stresses is shown. The failure is governed by competition between cracking and edge delamination of films and coatings under tension and between wrinkling, edge and buckle delamination under compression.

The failure of thin metal films and ceramic coatings induced by relaxation of intrinsic and extrinsic stresses is found to be controlled by essentially the same mechanisms. Which mechanism will be acting is determined by the correlation of mechanical characteristics of the film, the substrate and the interface.

The work is supported by RFBR under Grant No. 14-01-92005 HHC_a.

\section{REFERENCES}

1. L. B. Freund and S. Suresh, Thin Film Materials: Stress, Defect Formation and Surface Evolution (Cambridge University Press, Cambridge, 2003).

2. R. W. Hoffman, Thin Solid Films 34, 185 (1976).

3. P. Chaudhari, J. Vac. Sci. Tech. 9, 520 (1972).

4. R. C. Cammarata, T. M. Trimble, and D. J. Srolovitz, J. Mater. Res. 15, 2468 (2000).

5. C. Friesen and C. V. Thompson, Phys. Rev. Lett. 89, 126103-1-4 (2002).

6. E. Chason, B. W. Sheldon, L. B. Freund, J. A. Floro, and S. J. Hearne, Phys. Rev. Lett. 88, 156103-1-4 (2002).

7. F. Spaepen, Acta Mater. 48, 31 (2000).

8. F. M. d'Heurle, Metall. Trans. 1, 725 (1970).

9. M. F. Doerner and W. D. Nix, Crit. Rev. Solid State Mater. Sci. 14, 225 (1988).

10. H. H. Yu, M. Y. He, and J. W. Hutchinson, Acta Mater. 49, 93 (2001). 